\title{
Magnetic Structure of an Erupting Filament
}

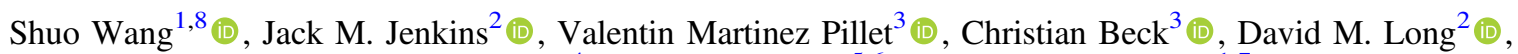 \\ Debi Prasad Choudhary $^{4}$ (D), Karin Muglach ${ }^{5,6}$ (iD), and James McAteer ${ }^{1,7}$ (D) \\ ${ }^{1}$ Department of Astronomy, New Mexico State University, P.O. Box 30001, MSC 4500, Las Cruces, NM 88003, USA; mcateer@nmsu.edu \\ ${ }^{2}$ Mullard Space Science Laboratory, University College London, Holmbury St. Mary, Dorking, Surrey, RH5 6NT, UK \\ ${ }^{3}$ National Solar Observatory, 3665 Discovery Drive, Boulder, CO 80303, USA \\ ${ }^{4}$ Department of Physics and Astronomy, California State University Northridge, Northridge, CA 91330-8268, USA \\ ${ }^{5}$ Catholic University of America, Washington, DC 20064, USA \\ ${ }^{6}$ NASA Goddard Space Flight Center, Greenbelt, MD 20771, USA \\ ${ }^{7}$ Sunspot Solar Observatory, Sunspot, NM 88349, USA \\ Received 2020 January 31; accepted 2020 February 5; published 2020 March 31
}

\begin{abstract}
The full 3D vector magnetic field of a solar filament prior to eruption is presented. The filament was observed with the Facility Infrared Spectropolarimeter at the Dunn Solar Telescope in the chromospheric He I line at $10830 \AA$ on 2017 May 29 and 30. We inverted the spectropolarimetric observations with the Hanle and Zeeman Light code to obtain the chromospheric magnetic field. A bimodal distribution of field strength was found in or near the filament. The average field strength was 24 Gauss, but prior to the eruption we find the 90th percentile of field strength was 435 Gauss for the observations on May 29. The field inclination was about $67^{\circ}$ from the solar vertical. The field azimuth made an angle of about $47^{\circ}-65^{\circ}$ to the spine axis. The results suggest an inverse configuration indicative of a flux rope topology. He I intensity threads were found to be coaligned with the magnetic field direction. The filament had a sinistral configuration as expected for the southern hemisphere. The filament was stable on 2017 May 29 and started to rise during two observations on May 30, before erupting and causing a minor coronal mass ejection. There was no obvious change of the magnetic topology during the eruption process. Such information on the magnetic topology of erupting filaments could improve the prediction of the geoeffectiveness of solar storms.
\end{abstract}

Unified Astronomy Thesaurus concepts: Quiet solar chromosphere (1986); Solar filaments (1495); Solar filament eruptions (1981); Solar magnetic fields (1503)

\section{Introduction}

Solar filaments are relatively thin, elongated, and magnetically bound structures observed in absorption when projected against the chromosphere of the solar disk. They are composed of cold $\left(10^{4} \mathrm{~K}\right)$, dense (electron density $>10^{9} \mathrm{~cm}^{-3}$ ) plasma which is thermally insulated from its much hotter surroundings in the transition region and corona (Parenti 2014). Filaments are always located above magnetic field polarity inversion lines in the photosphere. Depending on the magnetic conditions within the photosphere below, filaments may be categorized as either active, intermediate, or quiescent. Quiescent filaments are typically more extended (hundreds of $\mathrm{Mm}$ ), elevated (tens of $\mathrm{Mm}$ ), and long lived (months) than their active region counterparts, in addition to exhibiting a weaker host magnetic field (a few Gauss) (e.g., Mackay et al. 2010). Filaments are composed of fine threads which form an angle $\left(20^{\circ}-35^{\circ}\right)$ to their spine (Lin et al. 2007; Parenti 2014). Filaments and prominences can be observed in strong chromospheric spectral lines such as $\mathrm{H} \alpha$ at $6563 \AA$, or the absorption lines of He I at $5876 \AA$ A (He I D3) and $10830 \AA$ A. In particular, spectropolarimetry of $10830 \AA$ is a powerful tool used to study the magnetic structure in the upper atmosphere (e.g., Choudhary et al. 2002; Schad et al. 2013, 2015, 2016). Lin et al. (1998) demonstrated measurements of the full Stokes parameters of a filament at $10830 \AA$, and proposed a tilted magnetic field loop across the filament spine to explain their observations.

Casini et al. (2003) presented results of He I D3 inversions in a prominence, finding a range of magnetic field strengths of

\footnotetext{
${ }^{8}$ DKIST Ambassador
}

between 10 and 70 Gauss. Merenda et al. (2006) found magnetic field strengths of about 30 Gauss in a polar crown prominence using observations taken in the $\mathrm{He} \mathrm{I}$ line at $10830 \AA$ A. However, much larger field strengths have also been observed. For example, an active region filament with a magnetic field strength of up to 800 Gauss was observed on 2005 May 17 by $\mathrm{Xu}$ et al. (2012). The same filament was activated by a solar flare on 2005 May 18, and showed multiple components in the profiles (Sasso et al. 2011). Orozco Suárez et al. (2014) studied the magnetic field of a prominence, and found that the magnetic field was stronger in its feet than in its main body. However, the study of the magnetic field of prominences is not without its complications. For example, Milić et al. (2017) studied the multidimensional effects on the inference of the magnetic field of prominences, and found that the inferred magnetic field is weaker and more horizontal than their input. Furthermore, and quite crucially, Díaz Baso et al. (2019a, 2019b) studied an active region filament observed on 2014 June 17, and found that the Stokes $V$ signals were not from the filament, but from the active region below it.

In addition to the amplitude, inversions also provide information about the orientation of the filament's host magnetic field. Collados et al. (2003) studied the azimuth of two filaments close to the disk center, and found that it is oriented approximately along the filament axis. Merenda et al. (2007) studied the orientation of the magnetic field of a solar filament near the disk center, and found that the inclination of the magnetic field is horizontal in the central part of the filament and changes along the filament axis, and its azimuth changes along the filament as well. Hanaoka \& Sakurai (2017) 

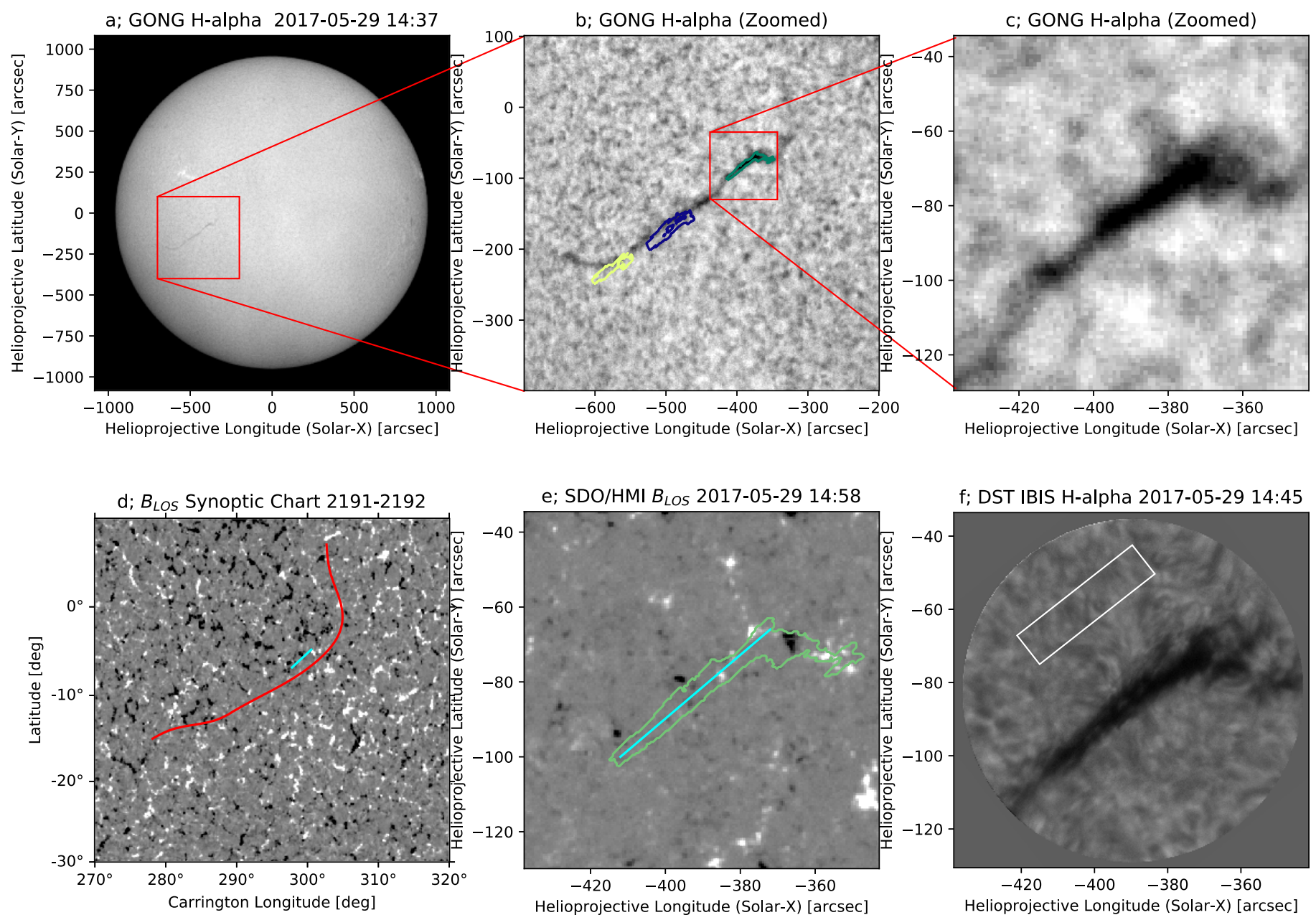

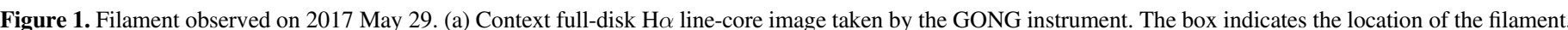

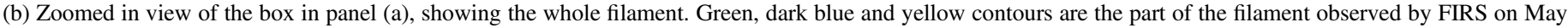

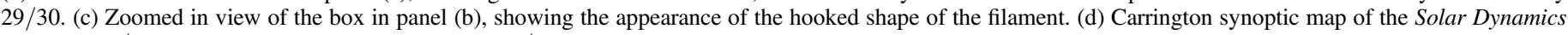

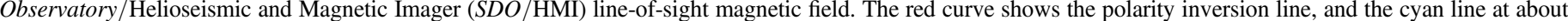

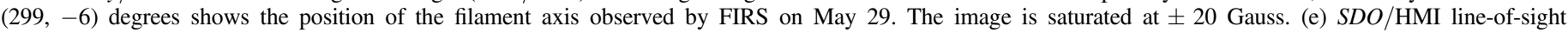

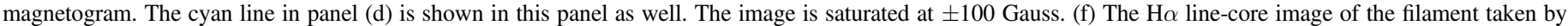
IBIS. The white box indicates the quiet-Sun area over which the average IBIS profile was calculated. Images observed by FIRS are aligned to $\sim 1^{\prime \prime}$.

statistically studied the orientation of the magnetic field of filaments and confirmed their hemispheric pattern of chirality.

After filament formation, filaments can be stable within the solar atmosphere for a few rotations. The stability of filaments is heavily dependent on the evolution of its host magnetic field; in the case of quiescent filaments it is believed their stability is additionally dependent on the filament plasma itself (see, e.g., Zirker et al. 1998; Török \& Kliem 2005; Kliem \& Török 2006; Berger et al. 2008; Fan 2018; Jenkins et al. 2018, 2019). When this stability is removed, some filament eruptions lead to coronal mass ejections (CMEs) (Prasad Choudhary et al. 2002; Schwenn 2006), while others do not (Choudhary \& Moore 2003). The magnetic structure of an active region filament observed on 2005 May 17 was explained as a rising flux rope by Sasso et al. (2014). Kuckein et al. (2009, 2012a, 2012b) studied an active region filament observed in 2005 July, and suggested a flux rope topology. Together, the field strengths and angles can be used to distinguish between the multiple topologies that were suggested to be able to support the filament plasma, such as the sheared arcade model and the flux rope model (Gibson 2018).
Regular full vector magnetic fields of solar filaments are now obtained from synoptic observations at the Dunn Solar Telescope (DST). In this article we present the inversion of He I $10830 \AA$ as observed within a filament during an observing campaign carried out in 2017 May. In addition, we present the first high-resolution observations in He I $10830 \AA$ acquired during a filament eruption along with the corresponding Hanle and Zeeman Light (HAZEL; Asensio Ramos et al. 2011) inversion results. The line-of-sight velocity information will be used in a future paper. Section 2 describes our data sets. The analysis with the HAZEL code is detailed in Section 3. Our results are presented in Section 4 and discussed in Section 5. Section 6 gives our conclusions.

\section{Observations}

A quiescent filament, shown in Figure 1, was observed ondisk $\left(-24^{\circ} .3,-4.85\right)\left(\left[x:-500^{\prime \prime}, y:-200^{\prime \prime}\right]\right)$ at $14: 44: 55 \mathrm{UT}$ on 2017 May 29. Figure 1(a) shows a full-disk context image taken in the $\mathrm{H} \alpha$ line core at $6562.8 \AA$ using the Global 
Oscillation Network Group (GONG) ${ }^{9}$ (Harvey et al. 2011). Figure 1(b) zooms in on the box in Figure 1(a) to show the filament of interest, this is then further zoomed in to the field of view (FOV) of the ground-based instruments at the DST in Figure 1(c). Simultaneous photospheric magnetic field information (Figure 1(d)) shows the cospatial photospheric polarity inversion line. Figure 1(f) shows the filament as observed using the Interferometric Bidimensional Spectropolarimeter (IBIS; Cavallini 2006) mounted at the DST. In addition to IBIS, observations were taken using the Facility Infrared Spectropolarimeter (FIRS; Jaeggli et al. 2010), the Rapid Oscillations in the Solar Atmosphere (Jess et al. 2010), and the Spectropolarimeter for Infrared and Optical Regions (SPINOR; SocasNavarro et al. 2006). The coudé table was rotated such that the slits of FIRS and SPINOR were aligned with the axis of the filament. We focus only on the observations taken with IBIS and FIRS here. Equipped with a high-order adaptive optics system (see Rimmele 2004 for a detailed description), the DST is capable of capturing diffraction-limited images of the Sun at high temporal cadence. The filament was observed at the DST for several days prior to its eruption, occurring on the day following the snapshot shown in Figure 1. The length of the filament including the additional portion of the filament that extends towards the northern hemisphere (note the filament channel can be seen in the extreme ultraviolet observations) is $660^{\prime \prime}$. Due to the total length of the filament of $660^{\prime \prime}$ and the limited FOV at the DST, only one of the ends of the filament and its surroundings were captured in the ground-based observations.

IBIS scanned across the two lines of $\mathrm{H} \alpha$ at $6562.8 \AA$ and $\mathrm{Ca}$ II IR at $8542 \AA$ in spectroscopic mode with a nonequidistant spectral sampling of 27 and 30 wavelength points, respectively. The exposure time was $80 \mathrm{~ms}$ per image with a total cadence of $13 \mathrm{~s}$ for both lines. A circular FOV with a diameter of $95^{\prime \prime}$ was sampled with 0 ". 1 pixel $^{-1}$ in both $x$ and $y$. Data were acquired from 13:59:46 to 22:18:10 UT on May 29, with a gap between 15:42:12 and 20:59:00 UT, and from 13:47:08 to 15:02:13 UT on May 30. The IBIS data were reduced with the standard data reduction pipeline. ${ }^{10}$ In addition to the standard processing, the influence of the prefilter transmission curve on the line profiles was removed by forcing the average quiet-Sun profile, defined over the region bound by the square box shown in Figure 1(f), into the shape of a reference profile given by the tables of David (1961) at $\mu=0.91$ (for more details see Schwartz et al. 2019). The correction retrieved for the average profile was applied to all other spectra. This step also provides an radiometric intensity calibration.

FIRS was used to observe the photospheric Si I $10827 \AA$ line and the chromospheric He I $10830 \AA$ line using a $40 \mu \mathrm{m}$ wide $\left(\approx 0\right.$." 3 ), single slit of $75^{\prime \prime}$ length that was sampled with 0 " 15 pixel $^{-1}$ along the slit. A spectral range from 10817 to $10856 \AA$ was covered with a spectral sampling of $0.039 \AA$ pixel $^{-1}$. The exposure time was $125 \mathrm{~ms}$ with a total integration time of $20 \mathrm{~s}$ (8 s) for the four Stokes parameters per scan step on May 29 (May 30). The noise rms in continuum windows in Stokes $I$ of raw data was $1 \times 10^{-2} / 3 \times 10^{-2} / 6 \times 10^{-2}$ for the three observations on May 29 and 30. The filament was scanned with 100 (200) steps of 0." 3 step width on May 29 (May 30) with a total duration of 40 minutes (30 minutes). Data were acquired

\footnotetext{
9 http://halpha.nso.edu/index.html

10 https://www.nso.edu/wp-content/uploads/2018/05/ibis_tn_005.pdf
}

from 14:41:33 to $15: 21: 30$ UT on May 29 and 13:46:58 to 15:02:14 UT on May 30. The FIRS data were reduced with the standard data reduction pipeline. ${ }^{11}$ In addition to the standard processing, we ran a two-dimensional Fourier filter to reduce polarized interference fringes and a despiking routine to capture hot pixels over the FIRS spectra. Prior to the inversion, the data was rebinned to 0 ! $9 \times 0$ ". 9 per pixel to improve the signal-tonoise ratio. The final noise rms in continuum windows was $8 \times 10^{-3}$ of $I_{c}$ and $4 \times 10^{-4}$ of $Q_{c}, U_{c}$, and $V_{c}$ for the May 29 and $2 \times 10^{-2} / 3 \times 10^{-2}$ of $I_{c}$, and $1 \times 10^{-3} / 1 \times 10^{-3}$ of $Q_{c}$, $U_{c}$, and $V_{c}$ for the first/second May 30 FIRS data.

Figure 2 displays overview maps of the three FIRS observations in the He I line. The filament exhibits a more compact structure on May 29 than on May 30 when several separate threads are visible. The barbs go in the counterclockwise direction, which implies a sinistral filament (Martin 1998). We find significant linear polarization signal inside the filament body in all maps. The circular polarization signal is concentrated toward the end of the filament at the right-hand side of the FOV in these maps on May 29. The filament was erupting on May 30. We traced individual threads in the $\mathrm{He}$ I line depth maps on May 30 as an independent estimate of the direction of the magnetic field azimuth. Figure 3 shows the Stokes images of Si $10827 \AA$ line from the photosphere. The photospheric magnetic field in the FOV is not relevant for the filament apart from the endpoint. The sequence of whiteblack-white patches at $x \sim 55^{\prime \prime}-70^{\prime \prime}$ corresponds to those found in the HMI magnetogram in Figure 1 at $x \sim-380^{\prime \prime}$ to $-360^{\prime \prime}, y=-70^{\prime \prime}$.

Additional context imagery of the filament and its surroundings was also supplied by the Atmospheric Imaging Assembly (AIA; Lemen et al. 2012) on the SDO (Pesnell et al. 2012) and the Extreme Ultraviolet Imager Telescope (EUVI; Wuelser et al. 2004) on board the Solar Terrestrial Relations Observatory Ahead (STEREO-Ahead; Kaiser et al. 2008).

\section{Inversion with HAZEL}

\subsection{Inversion Setup}

The HAZEL (Asensio Ramos et al. 2011) inversion code was used to infer the chromospheric magnetic field vector from the observations. The code requires full Stokes vector data, observation angle, and an initial estimate as input.

On May 24, the filament appeared at the solar east limb from Earth as shown in the left and middle columns in Figure 4. The filament was seen to have spanned between $5^{\prime \prime}$ and $50^{\prime \prime}$ above the limb, as observed by both the AIA and GONG instruments, in addition to a clear axis seen at $20^{\prime \prime}$. At its highest, it extended to a height of about $80^{\prime \prime}$ above the limb, according to observations taken by AIA in the $304 \AA$ passband, while the axis remained at 20". On May 27, the filament appeared at the solar west limb when observed by the STEREO-Ahead. This is shown in the upper right panel of Figure 4; its height remains similar to that observed on May 24. According to these observations the filament was stable between May 24 and 29, therefore we have used a fixed height of $20^{\prime \prime}$ above the solar surface in the inversion of the observation on May 29. Based on the line-of-sight velocity information of the two observations on May 30 we have estimated the distance traveled during the eruption process, providing height estimates of $46^{\prime \prime}$ and

\footnotetext{
$\overline{11}$ https://www.nso.edu/wp-content/uploads/2018/05/firs_soft_manual.pdf
} 


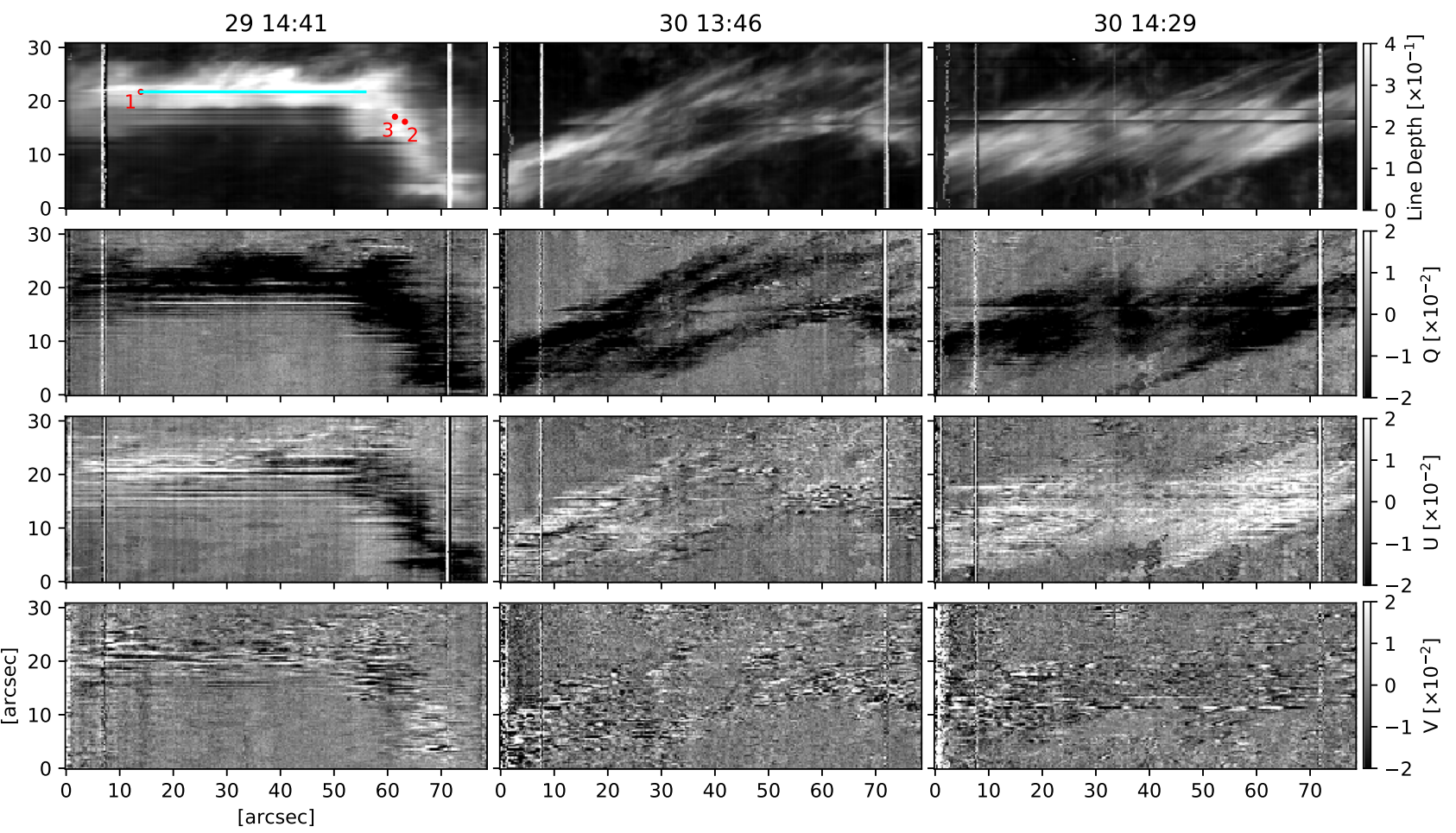

Figure 2. Spatial maps from the He I line red core observed by FIRS. From top to bottom: line depth, Stokes $Q, U$, and $V$ signals integrated over wavelength. Only the red lobe of the two He lines is integrated over wavelength for Stokes $V$. From left to right: 14:41 on 2017 May 29, 13:46 and 14:29 on May 30. The cyan line in the top left panel shows the position of the slit spectra in Figure 6. The three red dots in the top left panel shows the position of Stokes profiles in Figure 5.
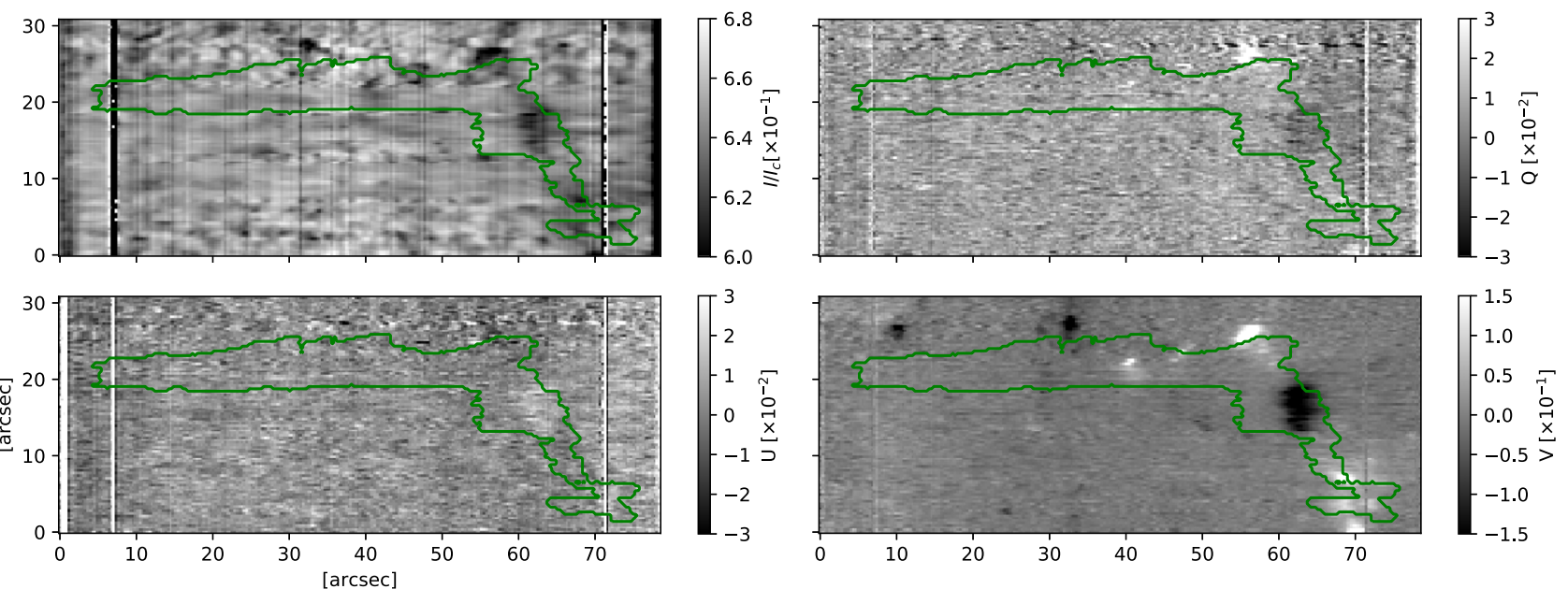

Figure 3. Spatial maps from the Si I $10827 \AA$ line observed by FIRS at 14:41 on 2017 May 29. Green contours show the observed filament in He I $10830 \AA$.

$109^{\prime \prime}$, respectively, for the inversion of the two data sets on May 30. Asensio Ramos et al. (2008), Schad et al. (2013), and Díaz Baso et al. (2019b) demonstrated that inversion results are not very sensitive to the specific value of height used, so these estimates are sufficient for our analysis.

The line profiles from our data in Figure 5 show only one dominant component, examples of the more complex multiple component line profiles can be found in Sasso et al. (2011). As the filament that is the focus of this study is far from any active region and the Stokes $V$ signals are much smaller than the linear polarization signals in most of the filament as shown in Figure 2, we may choose a single slab model for the inversion setup.

The inversion mode of DIRECT + Levenberg-Marquardt was selected in HAZEL to avoid getting trapped in a local minimum of the merit function. As a result, the initial guess does not have a large influence on the results (see Section 4.2 of Asensio Ramos et al. 2011 for more details). The seven output parameters of HAZEL are the magnetic field strength $B$, inclination $\gamma$, azimuth $\Phi$, optical thickness, thermal velocity, damping, and Doppler velocity. We focus here on the magnetic field parameters $B, \gamma$, and $\Phi$. A filament mask was obtained using the dark features in the FIRS Stokes $I$ line-core image of He I red.

\subsection{Disambiguation}

The inversion provides four different solutions that yield identical spectra because of the $180^{\circ}$ ambiguity and the Van Vleck ambiguity. The field direction has to therefore be 

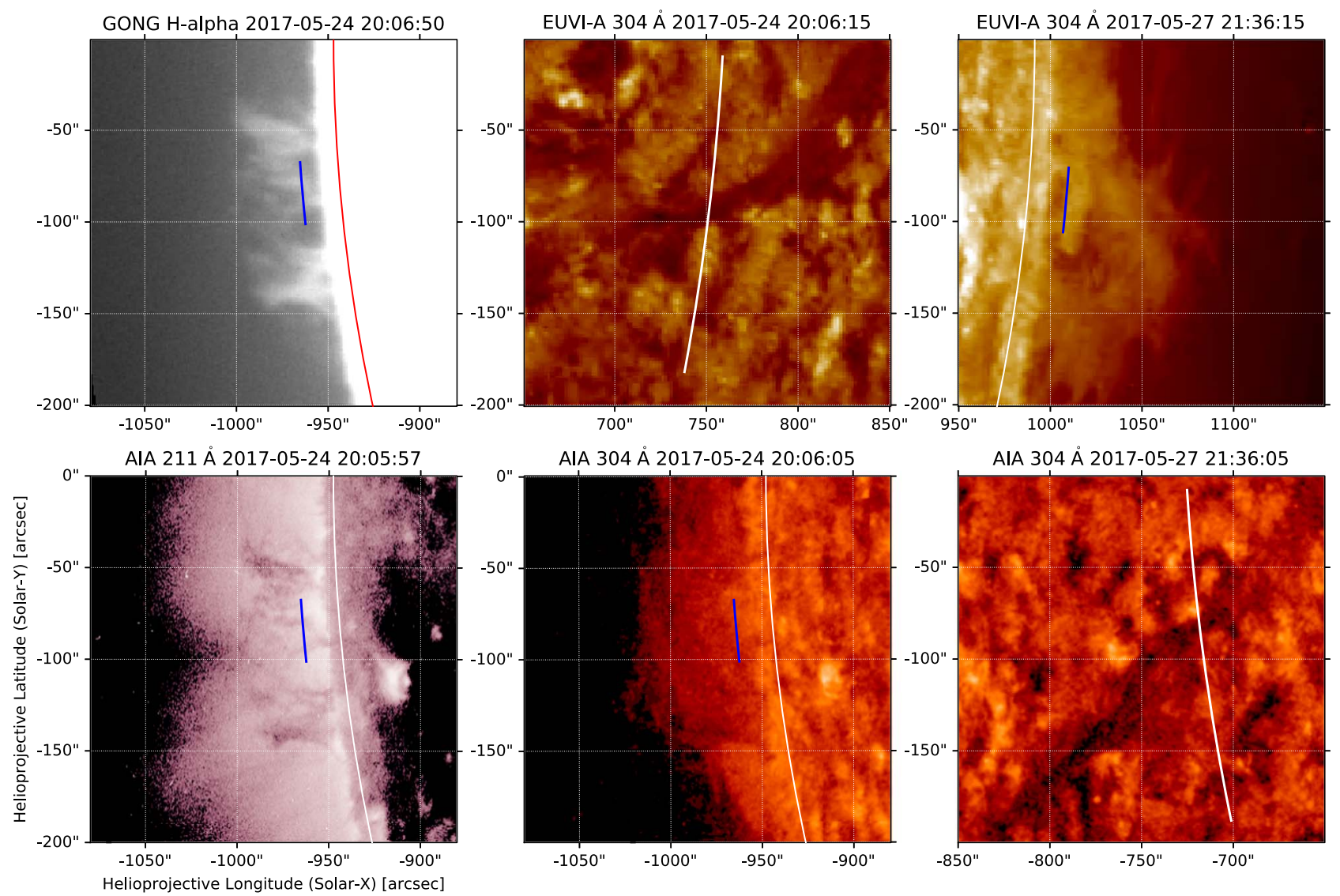

Figure 4. The filament observed in emission as a prominence at UT 20:06 on 2017 May 24 and UT 21:36 on 2017 May 27. Upper left: GONG H $\alpha$ map at UT 20:06 on 2017 May 24. Lower left: AIA $211 \AA$ map at UT 20:06 on 2017 May 24. Upper middle: STEREO-Ahead/EUVI 304 A map at UT 20:06 on 2017 May 24. Lower middle: SDO/AIA $304 \AA$ A map at UT 20:06 on 2017 May 24. Upper right: STEREO/EUVI $304 \AA$ map at UT 21:36 on 2017 May 27 . Lower right: SDO/AIA $304 \AA$ map at UT 21:36 on 2017 May 27. The white line in the upper middle panel shows the limb observed from Earth. The white line in the lower right panel shows the limb observed from STEREO-Ahead. The red/white lines in the other panels show the position of the solar limb. The blue lines show the height of $20^{\prime \prime}$ used in the inversion.

disambiguated using additional a priori information. Two of the solutions included an angle between field lines and the horizontal direction larger than $60^{\circ}$. Based on the results of previous studies (e.g., Trujillo Bueno et al. 2002) in which the field was found largely horizontal with small deviations, we discarded such solutions as unphysical. Furthermore, the leading polarity was positive as shown in the synoptic map of line-of-sight magnetic field (see Figure 1(d)). Thus, the magnetic field direction along the filament spine is from northwest to southeast i.e., the solution adopted appears to point the axial field in the direction compatible with the observed shear and the tilt angle from Joy's law.

\subsection{Fit Quality}

Figure 5 shows the inversion results of one position with a strong field and one with a weak magnetic field along the filament spine. The upper panels of Figure 5 show the inversion results of the pixel at $\left(x: 14^{\prime \prime}, y: 22^{\prime \prime}\right)$ in the spine of the observed filament (red point number 1 on the top left panel of Figure 2). The inverted field strength, inclination, and azimuth are 32 Gauss, $87^{\circ}$, and $-120^{\circ}$, respectively. The panels in the middle row of Figure 5 show the inversion results of the pixel at $\left(x: 63^{\prime \prime}, y: 16^{\prime \prime}\right)$ in the spine of the observed filament (red point number 2 on the top left panel of Figure 2). The panels in the lower row of Figure 5 show the inversion results of the pixel at $\left(x: 61^{\prime \prime}, y: 17^{\prime \prime}\right)$ in the spine of the observed filament (red point number 3 on the top left panel of Figure 2). The field strength, inclination, and azimuth are 577 Gauss, $91^{\circ}$, and $-92^{\circ}$, respectively. The rms of error and standard deviation of error of inverted magnetic field strength, inclination, and azimuth provided by HAZEL are listed in Table 1. They are derived from all the 1068 data points. The reversed shape of the Stokes $U$ profiles between the two positions in Figure 5 is mainly due to the large difference of azimuth. The signal of Stokes $V$ profiles in the middle and lower panels of Figure 5 are stronger than in the upper panels, which indicates a stronger line-of-sight magnetic field. Figure 6 shows the fit quality over multiple spectra, and the variation in $Q, U$, and $V$ along the cyan line in Figure 2. The observations are well reproduced with a single-component model throughout all of the filament.

\section{Results}

\subsection{Magnetic Field Strength}

Figure 7 shows the vector magnetic field of the observed filament. On May 29, the filament was stable. Strong magnetic fields were concentrated at the west end of the filament $(x>51)$ at the right-hand side of the FOV, where the 90th percentile of field strength was 435 Gauss. The mean value at $x<51^{\prime \prime}$ is 24 Gauss. A histogram of the field strength is shown in Figure 8. 

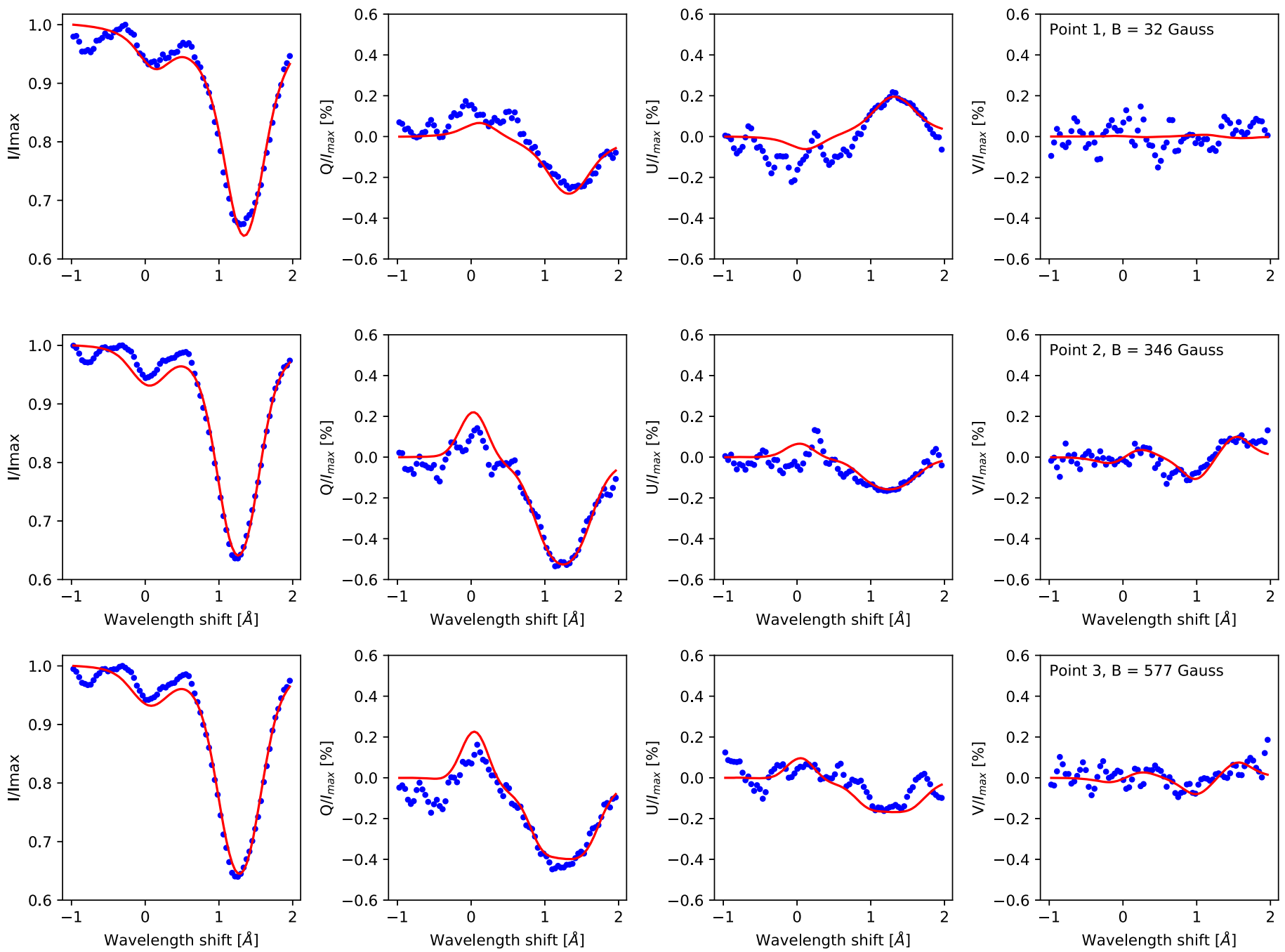

Figure 5. Stokes profiles of He I $10830 \AA$ A observed on 2017 May 29. Left to right: Stokes $I, Q, U$, and $V$. The upper/middle/lower panels correspond to the three numbered positions marked in Figure 2 by red dots. Blue dots are the observations. Red curves are best-fit lines from HAZEL. Wavelength shift is from 10829.0911 А̊.

Table 1

RMS of Error and Standard Deviation of Error of Inverted Magnetic Fields

\begin{tabular}{lccc}
\hline \hline Observation Time & Field Strength (Gauss) & Inclination (degrees) & Azimuth (degrees) \\
\hline 2017 May 29 14:41 & $0.5 \pm 0.4$ & $4.7 \pm 2.7$ & $5.8 \pm 3.9$ \\
2017 May 30 13:46 & $2.6 \pm 2.2$ & $11.1 \pm 8.5$ & $15.4 \pm 12.3$ \\
2017 May 30 14:29 & $3.9 \pm 3.5$ & $19.5 \pm 16.5$ & $21.2 \pm 15.0$ \\
\hline
\end{tabular}

The linear polarization of the filament in the He I $10830 \AA$ was strong, while there was almost no linear polarization in the Si I $10827 \AA$ line below the filament body, except the end. The structures of the horizontal magnetic fields are visible in the He I layer, but there is no indication in the Si I layer. Unlike linear polarization, the circular polarization at the end of the filament in the He I $10830 \AA$ shows a similar structure to the circular polarization in the Si I $10827 \AA$ below it, and does not show any similarity with the filament shape. The magnetic field strength in the end region of the filament reaches up to 570 Gauss in the HAZEL inversion, while below it in the photosphere, the field strength from HMI is only up to 340 Gauss. Possible reasons for the higher field strength derived from He I are that the photospheric value from the MilneEddington inversion of the HMI data is the average over the resolution element because of its spatial and spectral resolution, and filling factor impact. The magnetic field strength at the end is much stronger than in the filament spine, and it is consistent with the assumption that the filament goes down to the photosphere at the end.

On May 30, the filament started rising at $\sim 08: 00$. The first FIRS observation on this day was taken from 13:46 until 14:20, while the filament was rising. The part of the filament observed by FIRS at this time is shown in Figure 1(b) as a dark blue contour. The filament width on May 30 was about $12^{\prime \prime}$, which was larger than its width of $6^{\prime \prime}$ on May 29 (see Figure 7). The filament is not visible in the IBIS $\mathrm{H} \alpha$ line-core images on this day, but showed up as a strongly blueshifted line satellite. On May 30, the mean field strength value for the observation at 13:46 was 70 Gauss. The magnetic field strength was set to be 

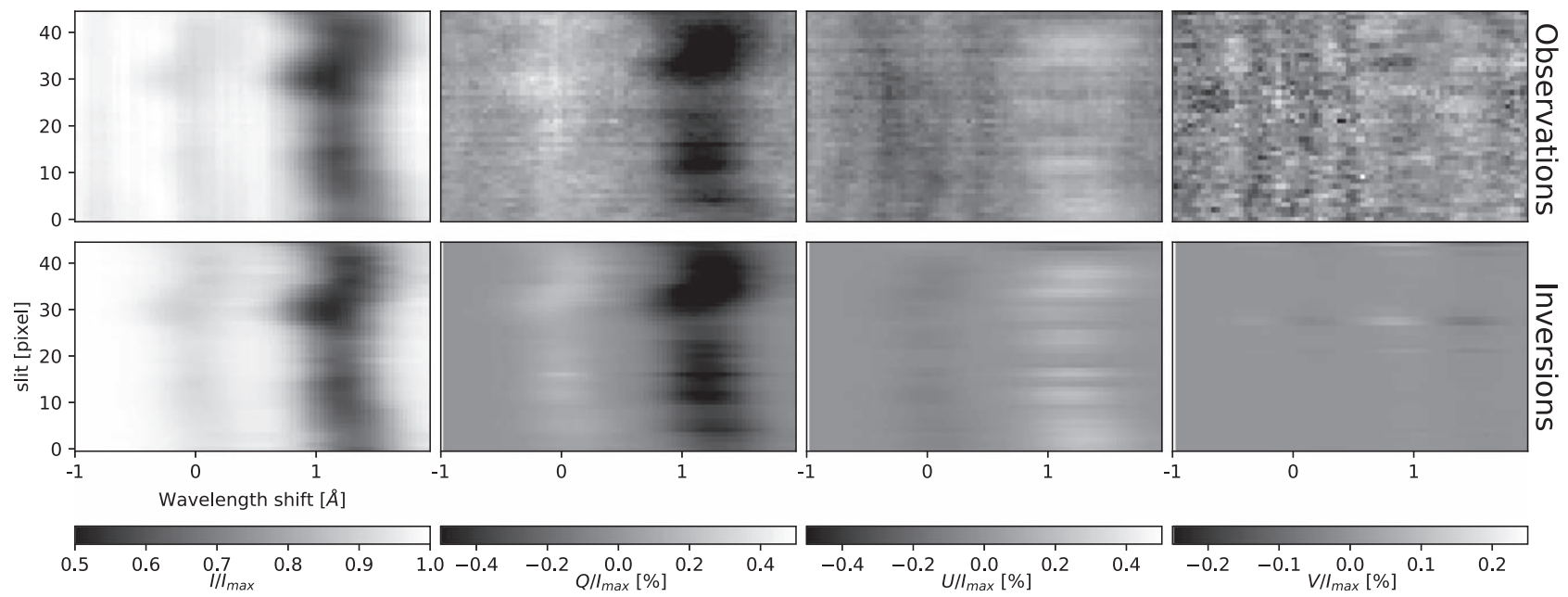

Figure 6. Top panels: observed slit spectra of Stokes $I, Q, U$, and $V$. Bottom panels: best-fit slit spectra of Stokes $I, Q, U$, and $V$. Wavelength shift is from $10829.0911 \AA$.
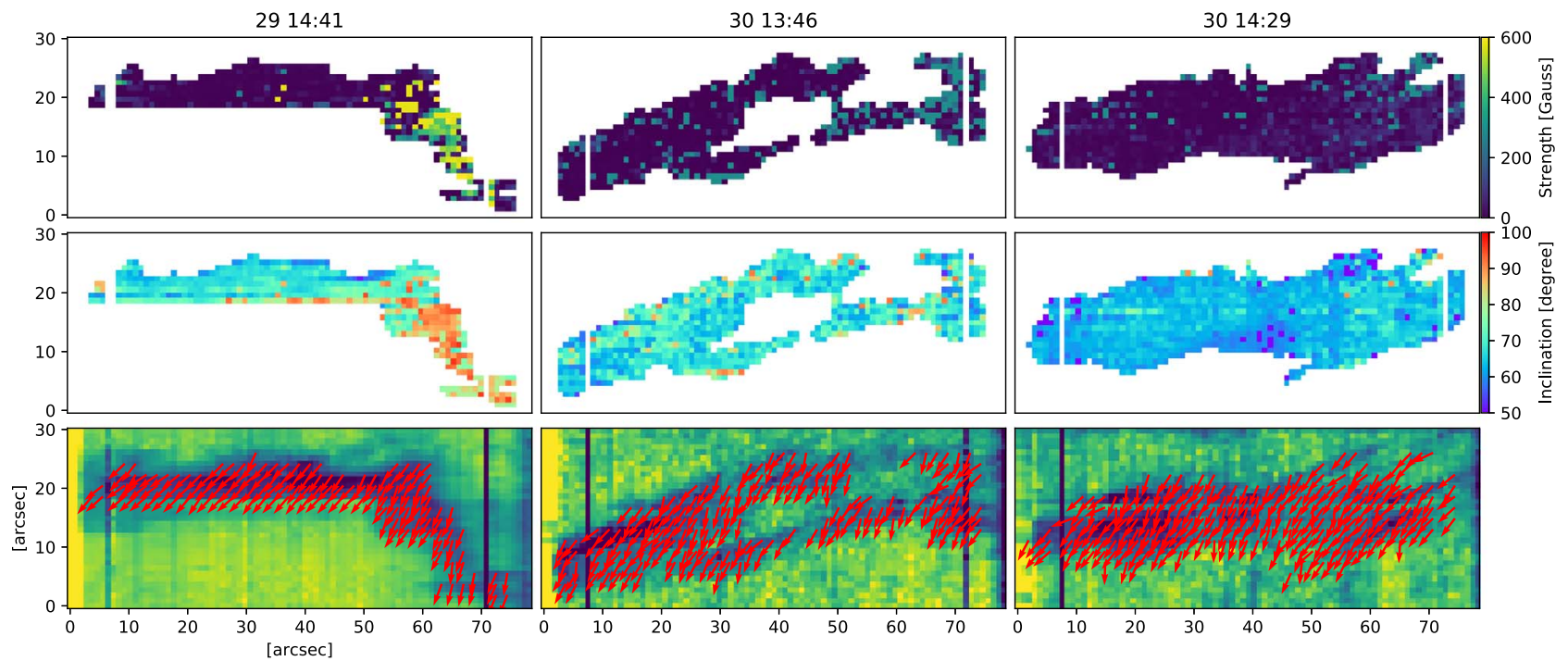

Figure 7. Vector magnetic field of the filament observed at 14:41 UT on 2017 May 29. Top row: magnetic field strength. Middle row: inclination. Bottom row: azimuth shown as arrows. The background is a line-core intensity map of He I.

no more than 300 Gauss in the inversion for the observations on May 30. The values of field strength that are close to this limit can be inaccurate due to the decreased line depth of the rising filament and the weak signal of Stokes $V$ for filaments observed close to disk center. The mean optical depth decreased from 0.74 on May 29 to 0.33 on May 30. The rising filament on May 30 was wider than on May 29, and this may be the reason of decreased line depth and optical depth.

Shortly afterwards, a second observation was made starting from 14:29 until 15:02, while the filament was still rising. The mean value of magnetic field strength was 45 Gauss for this observation. The filament disappeared in the SDO/AIA $304 \AA$ image at $\sim 18: 00$, and erupted as a minor CME.

\subsection{Angles of Magnetic Field}

The middle row of Figure 7 shows the inclination results of the three observations. An inclination value of 0/90/180 is defined to be upward/horizontal/downward with respect to the solar surface. On May 29, the inclination angles at positions along the filament spine $(x<51)$ were around $67^{\circ}$ and similar to each other, while the inclination at the end of the filament was around $79^{\circ}$, which is closer to horizontal than the spine region. The histograms of the inclination angles on May 29 are shown in the left panels of Figure 8. The mean values of the magnetic field inclination angle are $80^{\circ}$ for $x>51^{\prime \prime}$, and $69^{\circ}$ for $x<51^{\prime \prime}$.

The values of inclination are uniform for the observation at 13:46 on May 30, as shown in Figure 7. The mean value of the inclination angle observed at $13: 46$ is $69^{\circ}$. The values of inclination are similar everywhere for the observation at 14:29 on May 30, as shown in Figure 7 . The mean value of the inclination angle is $63^{\circ}$ for this observation.

The helium threads shown in the top left panel of Figure 2 are aligned with the magnetic field vectors, and they are also aligned with the IBIS $\mathrm{H} \alpha$ threads in Figure 1(f).

As shown in Figure 7, the direction of the magnetic field lines of the filament is from the following polarity to the leading polarity. In Figure 1(d), the leading polarity is positive, and the azimuth of photospheric magnetic field is expected to be from the leading polarity to the following polarity. Hence, the inversion results are suggestive of an inverse configuration 

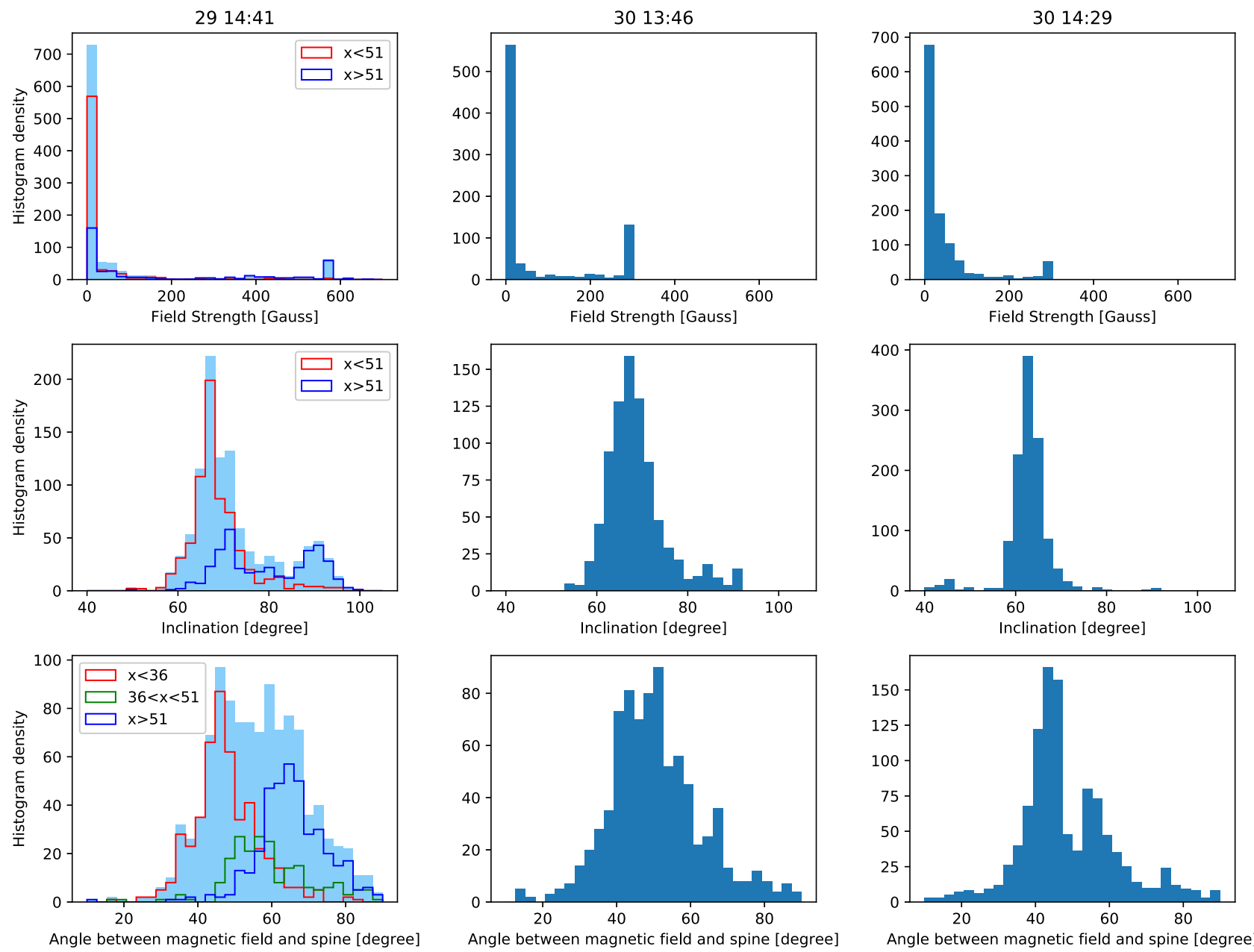

Figure 8. Top row: histogram of magnetic field strength. Middle row: histogram of inclination angle of magnetic field. Bottom row: histogram of angle between magnetic field and spine. From left to right: 14:41 on 2017 May 29, 13:46 and 14:29 on May 30. Positions indicated in the legend of the three left panels refer to positions of Figure 7.

of the magnetic field direction in the layer observed in $\mathrm{He} \mathrm{I}$ $10830 \AA$.

The lower left panel of Figure 8 shows the histogram of the angle between the magnetic field azimuth and the spine axis for the observation of May 29. The mean values of the angle between the magnetic field azimuth and the spine axis are $48^{\circ}$ for $x<36,59^{\circ}$ for $36<x<51$, and $66^{\circ}$ for $x>51$. The angles change gradually along the filament spine for the observation on May 29 . This value is $52^{\circ}$ for the observation at 13:46 on May 30, and was $54^{\circ}$ for the observation at 14:29 on May 30.

\section{Discussion}

The magnetic field results are in an inverse polarity configuration indicative of a flux rope topology. By inverse polarity configuration, we mean that the magnetic field vector crosses the polarity inversion line points from negative polarity to positive polarity. The polarity inversion line and photospheric polarities are shown in Figure 1(d). The pointing of the magnetic field of the filament is shown as azimuth in the bottom row of Figure 7. Figure 9 shows a sketch of this topology based on the observation of both the filament and the photospheric layers on May 29. The flux tube is completely above the photosphere with its bottom at the height of $20^{\prime \prime}$, where the filament lies. The diameter of the flux rope in
Figure 9 is assumed to be $70^{\prime \prime}$. Thus, each magnetic field line in the sketch makes about two turns (see the red arrows in Figure 9). The filament has a sinistral configuration as expected for the southern hemisphere.

We investigated the vector magnetic field of a quiescent filament from spectropolarimetric scans in the He I $10830 \AA$. Our results demonstrate an inverse polarity configuration indicative of a flux rope topology before the eruption process. The magnetic topology does not change during the initial eruption process.

The magnetic field strength of the filament is mainly 24 Gauss. The value is consistent with studies of quiescent filaments observed at the limb. For example, 20 Gauss from Casini et al. (2003), 7 Gauss from Orozco Suárez et al. (2014), and 60 Gauss from Martínez González et al. (2015). However, the magnetic field strength at the filament end is much stronger than the value at the central part, and the same trend is shown in studies at the limb (Orozco Suárez et al. 2014). The structures of the horizontal magnetic fields are visible in the He I layer, but there is no indication in the Si I layer. In contrast, AR filaments are usually observed with large scale horizontal structures in both the He I layer and the Si I layer (Kuckein et al. 2012b; Xu et al. 2012; Díaz Baso et al. 2019b). The magnetic field in the filament body seems completely detached from the photospheric field below it, and it is in line 


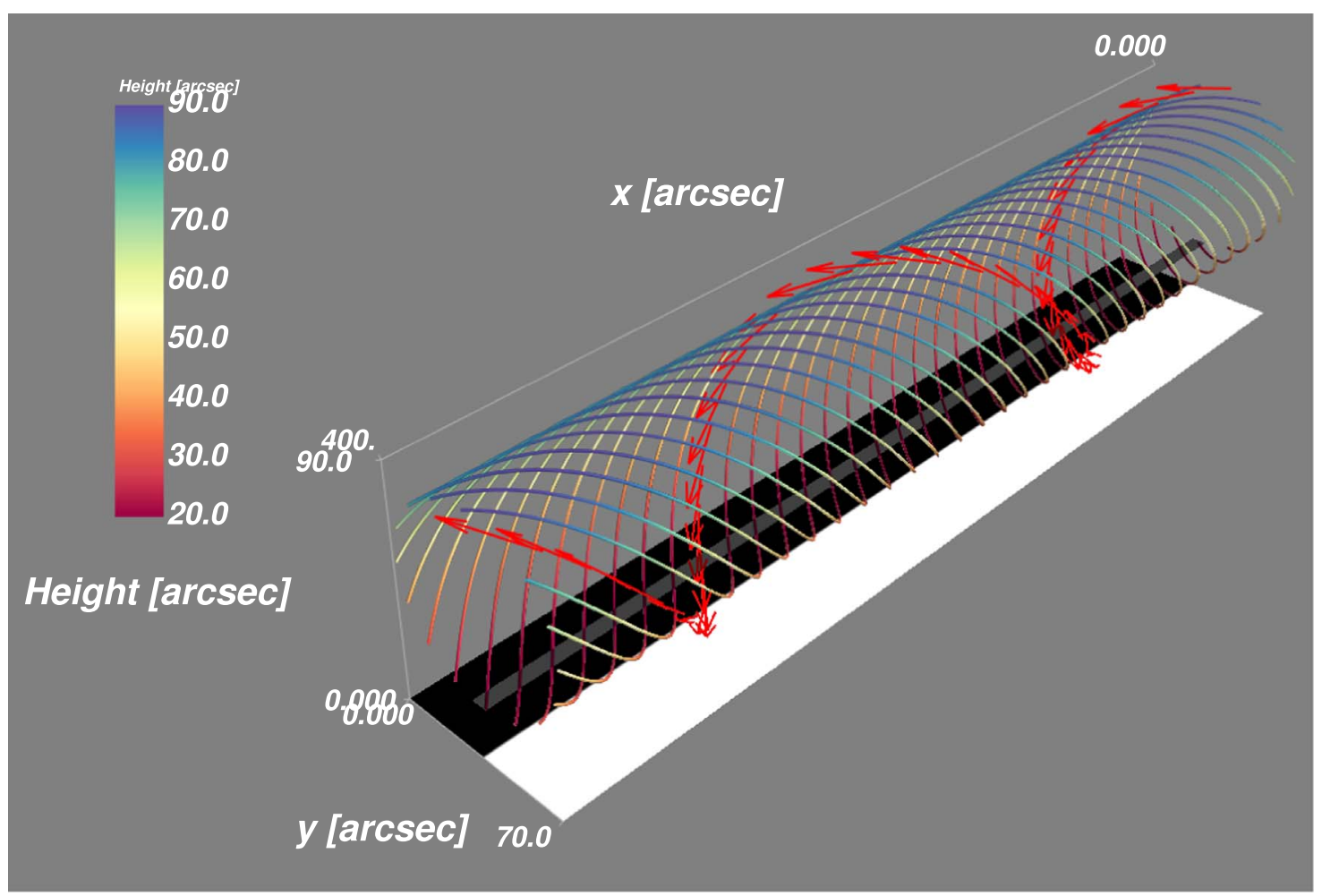

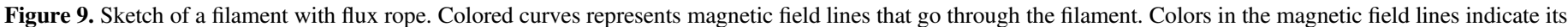

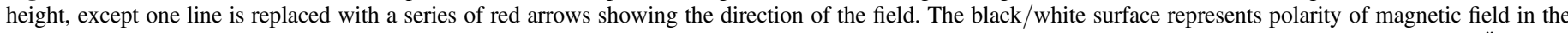
photosphere under the flux rope at height 0 . The gray surface represents the observed surface of the filament at the bottom of the flux rope (height of $20^{\prime \prime}$ ).

with the hyperbolic flux tube (Titov et al. 2002, 2003) interpretation of a flux rope, whereas AR filaments are believed to be low lying and therefore contain bald point separatrix surface signatures in the photosphere.

The inclination angle to the solar vertical is around $67^{\circ}$ for the observed part of the stable filament on May 29. Reported inclination of a quiescent prominence ranges widely from mostly horizontal (Casini et al. 2003) to $30^{\circ}$ from vertical (Martínez González et al. 2015), and $77^{\circ}$ from vertical (Orozco Suárez et al. 2014). The angle between magnetic field azimuth and filament spine is $47^{\circ}-58^{\circ}$, and gradually decreases along the filament from the end on May 29. Orozco Suárez et al. (2014) reported $58^{\circ}$ or $24^{\circ}$ for the angle of a quiescent prominence. In general, the polarimetric data can be used to determine the chirality of filaments without information about barb direction.

The flux rope topology was found on the day prior to the eruption, and during the eruption without any evident changes. The filament was observed on two consecutive days, and was rising while being observed on the second day. The inversions give the field strength for both days. During the eruption, the observed magnetic structure is well preserved without significant changes. The eruption itself does not seem to impact the observed field strengths significantly.

The quiescent filament has a length of about $660^{\prime \prime}$ and a width of about $6^{\prime \prime}$ in the $\mathrm{H} \alpha$ observation on 2017 May 29. The filament expanded and doubled its width on the second day, and the line depth decreased as shown in Figure 2. The line depth reduced on 2017 May 30 due to rising of the filament. These facts are consistent with the rise and expansion of the filament through the solar atmosphere. The filament spine was almost straight with both ends in an S-shape. It resembles the sigmoidal morphology observed by X-rays of active regions (Canfield et al. 1999).

\section{Conclusions}

We derived the magnetic field vector in a filament prior to and during its eruption process. The magnetic field topology indicates a flux rope. The field topology does not change significantly during the initial phase of the eruption. Using this information on the magnetic field of erupting material on the Sun could improve the prediction of the geoeffectiveness of the related solar storms. The synoptic observations that are currently run at the DST aim to provide suited observations for this purpose.

This work is funded by NSF 1839306. Sunspot Solar Observatory is a multi-institution consortium that is funded by multiple entities including NSF $(1649052,1945705)$ and the State of New Mexico. Funding for the DKIST Ambassadors program is provided by the National Solar Observatory, a facility of the National Science Foundation, operated under Cooperative Support Agreement number AST-1400405. J.M.J. thanks the STFC for support via funding given in his $\mathrm{PhD}$ studentship, and travel funds awarded by the Royal Astronomical Society. D.M.L. acknowledges support from the European Commission's H2020 Programme under the following grant agreements: GREST (No. 653982) and Pre-EST (No. 739500) as well as support from the Leverhulme Trust for an Early Career Fellowship (ECF-2014-792) and is grateful to the Science Technology and Facilities Council for the award of an Ernest Rutherford Fellowship (ST/R003246/1). D.P.C. was partially supported through NSF grant AGS-1413686. K.M. acknowledges support by the NASA Heliophysics Guest 
Investigator program and the NASA cooperative agreement NNG11PL10A.

\section{ORCID iDs}

Shuo Wang (i) https://orcid.org/0000-0001-5589-0416 Jack M. Jenkins (iD https://orcid.org/0000-0002-8975-812X Valentin Martinez Pillet (i) https://orcid.org/0000-00017764-6895

Christian Beck (iD https://orcid.org/0000-0001-7706-4158

David M. Long (iD https://orcid.org/0000-0003-3137-0277

Debi Prasad Choudhary (iD https://orcid.org/0000-0002-

9308-3639

Karin Muglach (iD https://orcid.org/0000-0002-5547-9683

James McAteer (1D https://orcid.org/0000-0003-1493-101X

\section{References}

Asensio Ramos, A., Trujillo Bueno, J., \& Landi Degl'Innocenti, E. 2008, ApJ, 683,542

Asensio Ramos, A., Trujillo Bueno, J., \& Landi Degl'Innocenti, E. 2011, HAZEL: HAnle and ZEeman Light, Astrophysics Source Code Library, ascl:1109.004

Berger, T. E., Shine, R. A., Slater, G. L., et al. 2008, ApJL, 676, L89

Canfield, R. C., Hudson, H. S., \& McKenzie, D. E. 1999, GeoRL, 26, 627

Casini, R., López Ariste, A., Tomczyk, S., et al. 2003, ApJL, 598, L67

Cavallini, F. 2006, SoPh, 236, 415

Choudhary, D. P., \& Moore, R. L. 2003, GeoRL, 30, 2107

Choudhary, D. P., Suematsu, Y., \& Ichimoto, K. 2002, SoPh, 209, 349

Collados, M., Trujillo Bueno, J., \& Asensio Ramos, A. 2003, in ASP Conf. Proc. 307, Solar Polarization, ed. J. Trujillo-Bueno \& J. Sanchez Almeida (San Francisco, CA: ASP), 468

David, K.-H. 1961, ZAp, 53, 37

Díaz Baso, C. J., Martínez González, M. J., \& Asensio Ramos, A. 2019a A\&A, 625, A129

Díaz Baso, C. J., Martínez González, M. J., \& Asensio Ramos, A. 2019b, A\&A, 625, A128

Fan, Y. 2018, ApJ, 862, 54

Gibson, S. E. 2018, LRSP, 15, 7

Hanaoka, Y., \& Sakurai, T. 2017, ApJ, 851, 130

Harvey, J. W., Bolding, J., Clark, R., et al. 2011, AAS/Solar Physics Division Abstracts, 42, 17.45
Jaeggli, S. A., Lin, H., Mickey, D. L., et al. 2010, MmSAI, 81, 763

Jenkins, J. M., Hopwood, M., Démoulin, P., et al. 2019, ApJ, 873, 49

Jenkins, J. M., Long, D. M., van Driel-Gesztelyi, L., et al. 2018, SoPh, 293, 7

Jess, D. B., Mathioudakis, M., Christian, D. J., et al. 2010, SoPh, 261, 363

Kaiser, M. L., Kucera, T. A., Davila, J. M., et al. 2008, SSRv, 136, 5

Kliem, B., \& Török, T. 2006, PhRvL, 96, 255002

Kuckein, C., Centeno, R., Martínez Pillet, V., et al. 2009, A\&A, 501, 1113

Kuckein, C., Martínez Pillet, V., \& Centeno, R. 2012a, A\&A, 542, A112

Kuckein, C., Martínez Pillet, V., \& Centeno, R. 2012b, A\&A, 539, A131

Lemen, J. R., Title, A. M., Akin, D. J., et al. 2012, SoPh, 275, 17

Lin, H., Penn, M. J., \& Kuhn, J. R. 1998, ApJ, 493, 978

Lin, Y., Engvold, O., Rouppe van der Voort, L. H. M., et al. 2007, SoPh, 246, 65

Mackay, D. H., Karpen, J. T., Ballester, J. L., et al. 2010, SSRv, 151, 333

Martin, S. F. 1998, SoPh, 182, 107

Martínez González, M. J., Manso Sainz, R., Asensio Ramos, A., et al. 2015, ApJ, 802, 3

Merenda, L., Trujillo Bueno, J., \& Collados, M. 2007, in ASP Conf. Ser. 368, The Physics of Chromospheric Plasmas, ed. P. Heinzel et al. (San Francisco, CA: ASP), 347

Merenda, L., Trujillo Bueno, J., Landi Degl'Innocenti, E., et al. 2006, ApJ, 642, 554

Milić, I., Faurobert, M., \& Atanacković, O. 2017, A\&A, 597, A31

Orozco Suárez, D., Asensio Ramos, A., \& Trujillo Bueno, J. 2014, A\&A, 566, A46

Parenti, S. 2014, LRSP, 11, 1

Pesnell, W. D., Thompson, B. J., \& Chamberlin, P. C. 2012, SoPh, 275, 3

Prasad Choudhary, D., Srivastava, N., \& Gosain, S. 2002, A\&A, 395, 257

Rimmele, T. R. 2004, ApJ, 604, 906

Sasso, C., Lagg, A., \& Solanki, S. K. 2011, A\&A, 526, A42

Sasso, C., Lagg, A., \& Solanki, S. K. 2014, A\&A, 561, A98

Schad, T. A., Penn, M. J., \& Lin, H. 2013, ApJ, 768, 111

Schad, T. A., Penn, M. J., Lin, H., et al. 2015, SoPh, 290, 1607

Schad, T. A., Penn, M. J., Lin, H., et al. 2016, ApJ, 833, 5

Schwartz, P., Gunár, S., Jenkins, J. M., et al. 2019, A\&A, 631, A146

Schwenn, R. 2006, LRSP, 3, 2

Socas-Navarro, H., Elmore, D., Pietarila, A., et al. 2006, SoPh, 235, 55

Titov, V. S., Galsgaard, K., \& Neukirch, T. 2003, ApJ, 582, 1172

Titov, V. S., Hornig, G., \& Démoulin, P. 2002, JGRA, 107, 1164

Török, T., \& Kliem, B. 2005, ApJL, 630, L97

Trujillo Bueno, J., Landi Degl'Innocenti, E., Collados, M., et al. 2002, Natur, 415,403

Wuelser, J.-P., Lemen, J. R., Tarbell, T. D., et al. 2004, Proc. SPIE, 5171, 111

Xu, Z., Lagg, A., Solanki, S., et al. 2012, ApJ, 749, 138

Zirker, J. B., Engvold, O., \& Martin, S. F. 1998, Natur, 396, 440 